\title{
Implementation and experimental evaluation of Mega-voltage fan-beam CT using a linear accelerator
}

\author{
Hao Gong', Shengzhen Tao', Justin D. Gagneur², Wei Liu², Jiajian Shen², Cynthia H. McCollough', \\ Yanle $\mathrm{Hu}^{2^{*}}$ and Shuai Leng ${ }^{1^{*}}$
}

\begin{abstract}
Background: Mega-voltage fan-beam Computed Tomography (MV-FBCT) holds potential in accurate determination of relative electron density (RED) and proton stopping power ratio (SPR) but is not widely available.

Objective: To demonstrate the feasibility of MV-FBCT using a medical linear accelerator (LINAC) with a 2.5 MV imaging beam, an electronic portal imaging device (EPID) and multileaf collimators (MLCs).

Methods: MLCs were used to collimate MV beam along z direction to enable a $1 \mathrm{~cm}$ width fan-beam. Projection data were acquired within one gantry rotation and preprocessed with in-house developed artifact correction algorithms before the reconstruction. MV-FBCT data were acquired at two dose levels: 30 and 60 monitor units (MUs). A Catphan 604 phantom was used to evaluate basic image quality. A head-sized CIRS phantom with three configurations of tissue-mimicking inserts was scanned and MV-FBCT Hounsfield unit (HU) to RED calibration was established for each insert configuration using linear regression. The determination coefficient $\left(R^{2}\right)$ was used to gauge the accuracy of HU-RED calibration. Results were compared with baseline single-energy kilo-voltage treatment planning CT (TP-CT) HU-RED calibration which represented the current standard clinical practice.

Results: The in-house artifact correction algorithms effectively suppressed ring artifact, cupping artifact, and CT number bias in MV-FBCT. Compared to TP-CT, MV-FBCT was able to improve the prediction accuracy of the HU-RED calibration curve for all three configurations of insert materials, with $R^{2}>0.9994$ and $R^{2}<0.9990$ for MV-FBCT and TP-CT HU-RED calibration curves of soft-tissue inserts, respectively. The measured mean CT numbers of blood-iodine mixture inserts in TP-CT drastically deviated from the fitted values but not in MV-FBCT. Reducing the radiation level from 60 to $30 \mathrm{MU}$ did not decrease the prediction accuracy of the MV-FBCT HU-RED calibration curve.
\end{abstract}

Conclusion: We demonstrated the feasibility of MV-FBCT and its potential in providing more accurate RED estimation.

Keywords: Dose calculation, Electron density, Mega-voltage CT, Radiation therapy

\footnotetext{
*Correspondence: Hu.Yanle@mayo.edu; Leng.Shuai@mayo.edu

1 Department of Radiology, Mayo Clinic, 200 First Street SW, Rochester, MN 55905, USA

${ }^{2}$ Department of Radiology, Mayo Clinic Arizona, 5881 East Mayo Blvd, Phoenix, AZ 85258, USA
}

\begin{abstract}
Background
Radiation therapy (RT) is one of the most common cancer treatment methods $[1,2]$. It is minimally invasive and has the flexibility of optimizing radiation dose distributions conformal to treatment targets. The adoption of pre-treatment volumetric imaging and multileaf collimators (MLCs) in RT has enabled highly conformal
\end{abstract} original author(s) and the source, provide a link to the Creative Commons licence, and indicate if changes were made. The images or other third party material in this article are included in the article's Creative Commons licence, unless indicated otherwise in a credit line to the material. If material is not included in the article's Creative Commons licence and your intended use is not permitted by statutory regulation or exceeds the permitted use, you will need to obtain permission directly from the copyright holder. To view a copy of this licence, visit http://creativecommons.org/licenses/by/4.0/. The Creative Commons Public Domain Dedication waiver (http://creativeco mmons.org/publicdomain/zero/1.0/) applies to the data made available in this article, unless otherwise stated in a credit line to the data. 
treatment plans, which deliver lethal radiation dose to tumor while sparing organs-at-risk (OARs) surrounding the tumor [3]. Effective RT treatment relies on accurate calculation of radiation dose distribution within patients. Minimizing deviation between the calculated and delivered doses is always beneficial as it provides better estimation of doses delivered to the target and OARs and allows us to correlate radiation doses with treatment outcomes more accurately [4-6].

In clinical practice, relative electron density (RED) or proton stopping power ratio (SPR) is required to calculate photon or proton dose distribution, respectively [79]. These quantities can be obtained from CT numbers using a calibration curve established during the commissioning. CT number is a measure of linear attenuation coefficient which reflects a combined effect of Compton scattering $(\propto R E D)$, coherent scattering $\left(\propto Z^{1.86}\right)$ and photoelectric $\left(\propto Z^{3.62}\right)$ interaction mechanisms [8]. It depends not only on RED but also on chemical composition (or effective atomic number). Currently, treatment planning is based on treatment-planning CT (TP-CT) using kilovoltage $\mathrm{X}$-ray beams. In the energy range of TP-CT, Compton scattering is the main interaction mechanism but photoelectric effect and coherent scattering are not negligible. Materials with different chemical compositions may have the same CT numbers but different REDs or proton SPRs (degeneracy effect). However, based on the calibration curve method, these materials are mapped to the same RED or proton SPR, causing errors in RED or proton SPR estimation.

Compared to photon therapy, proton therapy dose calculation is more sensitive to the degeneracy effect. As an extreme example, if we add $1 \mathrm{~cm}$ of bolus in the beam path proximal to the treatment target which is equivalent to overriding $1 \mathrm{~cm}$ of air $(\mathrm{HU}=-1000)$ to water $(\mathrm{HU}=0)$, it results in only $2-3 \%$ of reduction in calculated dose to the treatment target for photon therapy. This, however, can pull back the spread-out Bragg peak by $1 \mathrm{~cm}$ for proton therapy, causing significant reduction in calculated dose to the treatment target. The degeneracy effect, although small, cannot be neglected for proton therapy. Clinically, an extra margin $(\sim 3.5 \%$ of water equivalent depth) is usually used to compensate potential errors in proton SPR estimation, or proton range uncertainty.

To reduce proton range uncertainty, one possible solution is dual-energy CT (DE-CT). Another potential solution is MV-FBCT, which is less susceptible to the degeneracy effect (or chemical composition variation) compared to TP-CT. Of note, in our recent study about proton range uncertainty [10], we have demonstrated that: first, the HU to SPR calibration curve is comprised of two segments, i.e. HU to RED conversion and RED to proton SPR conversion; second, when $\mathrm{x}$-ray photon energy is within $\mathrm{kV}$ range, the proton range uncertainty mainly resides in the segment of HU to RED conversion. As we move to MV-FBCT, contributions from the photoelectric interaction $\left(\propto Z^{3.62}\right) /$ coherent scattering and the sensitivity to chemical composition variation drop substantially compared to TP-CT. As a result, MV-FBCT HU maintains a better linear relationship with RED compared to treatment-planning CT. Meanwhile, mapping of RED to proton SPR goes through a logarithm operation which significantly suppresses the effect of chemical composition variation. Therefore, a calibration curve mapping from MV-FBCT HU to proton SPR is supposed to be more robust to chemical composition variation and thus has the potential to reduce proton range uncertainty. In a previous study intended to use MVCT to reduce proton range uncertainty near large metal implants, Newhauser et al. demonstrated the superb linear relationship between MVCT HU and proton SPR [11].

As of today, DECT has been thoroughly investigated but MV-FBCT has not received sufficient attention. A practical challenge of MV-FBCT is its availability. Currently, MV-FBCT is only available on Tomotherapy and Accuray Radixact (the next generation of TomoTherapy) systems. Yet, MV-FBCT in these systems is primarily designed for the purpose of patient setup. Use of these systems for treatment planning may be good enough for photon therapy as it is quite insensitive to chemical change variation, but may not be sufficient for proton therapy. In addition, these systems may not be available to many cancer centers.

The purpose of this work was to develop a method to acquire MV-FBCT images using the MV imaging beam, EPID, and MLC of a LINAC to improve the availability of MV-FBCT for the purpose of treatment planning. In addition, we developed an in-house data preprocessing pipeline to correct MV-FBCT image artifacts and CT number deviation caused by defects in raw projection data. We also assessed image quality of MV-FBCT and evaluated the prediction accuracy of the MV-FBCT HURED calibration curve compared to TP-CT. Even though Varian TrueBeam LINAC was used to demonstrate the feasibility of MV-FBCT in this study, the proposed method may be extended to other LINACs as well. With increased availability of MV-FBCT, we may stimulate more activities in exploring the potential of using MVFBCT to reduce proton range uncertainty.

\section{Methods}

Data acquisition

A standard TrueBeam LINAC (Varian Medical Systems, Palo Alto, California, USA) was used to acquire MVFBCT, using a $2.5 \mathrm{MV}$ imaging beam, a $43 \times 43 \mathrm{~cm}^{2}$ EPID 
Table 1 The parameters of imaging geometry and scanning protocols in MV-FBCT

\begin{tabular}{ll}
\hline Peak X-ray energy & $2.5 \mathrm{MeV}$ \\
Source-to-isocenter distance & $100 \mathrm{~cm}$ \\
Source-to-detector distance & $150 \mathrm{~cm}$ \\
Detector matrix size & $1280 \times 1280$ \\
Detector pixel size & $0.0336 \mathrm{~cm}$ \\
Scanning FOV (at iso-center) & $28.6 \mathrm{~cm}$ \\
Width of MLC slit (at iso-center) & $1.0 \mathrm{~cm}$ \\
Radiation dose (in delivered machine unit) & $30 \& 60 \mathrm{MU}$ \\
Number of projection per revolution & 306
\end{tabular}

Table 2 The configurations of insert materials

\begin{tabular}{lll}
\hline Config \# 1 (Gammex inserts) & $\begin{array}{l}\text { Config \#2 (CIRS } \\
\text { low-density } \\
\text { inserts) }\end{array}$ & $\begin{array}{l}\text { Config \#3 (CIRS } \\
\text { bone inserts) }\end{array}$ \\
\hline Adipose & Adipose & Bone $200 \mathrm{mg} / \mathrm{cc}$ \\
Brain & Breast 50/50 & Bone $800 \mathrm{mg} / \mathrm{cc}$ \\
Blood + lodine (2 mg/cc) & Liver & Bone $1000 \mathrm{mg} / \mathrm{cc}$ \\
Blood + lodine (4 mg/cc) & Lung (Exhale) & Bone $1250 \mathrm{mg} / \mathrm{cc}$ \\
Blood & Lung (Inhale) & Bone $1500 \mathrm{mg} / \mathrm{cc}$ \\
Breast 50/50 & Muscle & Bone $1750 \mathrm{mg} / \mathrm{cc}$ \\
Liver & Plastic water & \\
Solid water & & \\
\hline
\end{tabular}

placed $1.5 \mathrm{~m}$ from the source. An MLC was collimated to an in-plane field-of-view (FOV) of $28.7 \mathrm{~cm}$ and a crossplane width of $1.0 \mathrm{~cm}$ at the isocenter. The axial scan trajectory was used in data acquisition, with 306 projections per revolution. Projection data was acquired in a full rotation and MV-FBCT images were reconstructed using a standard FDK algorithm with the Hanning filter after correction of X-ray scatter, data truncation, beam-hardening, and ring-artifact. MV-FBCT data was acquired at two radiation dose levels of 30 and 60 MUs. A Catphan (Model 604, The Phantom Laboratory, Salem, New York, USA) was scanned to evaluate image quality. A headsized CIRS phantom (Model 062 M, CIRS, Norfolk, Virginia, USA) with three configurations of tissue-mimicking CIRS inserts and Gammex inserts (Sun Nuclear, Melbourne, Florida, USA) was used to evaluate HU value accuracy. Table 1 listed parameters used in MV-FBCT data acquisition. Table 2 listed all insert materials in each configuration. For both phantoms, the reconstructed FOV was $20 \mathrm{~cm}$ in diameter, the slice thickness and increment were both $0.1 \mathrm{~cm}$ for the high resolution module of the Catphan phantom and both $0.3 \mathrm{~cm}$ for all other cases.

To demonstrate that MV-FBCT HU maintains a better linear relationship with RED compared to TP-CT HU, we also acquired TP-CT images of tissue-mimicking inserts using the same phantom setups on a commercial TP-CT simulator (Somatom Definition AS, Siemens Medical Solution USA, Malvern, PA, USA). Key imaging parameters used in TP-CT included $120 \mathrm{kVp}, 200 \mathrm{mAs}, 0.8$ pitch, $30 \mathrm{~cm}$ reconstructed FOV, and $0.3 \mathrm{~cm}$ slice thickness and increment.

\section{Artifact correction in projection domain}

An in-house preprocessing pipeline was implemented to suppress MV-FBCT image artifacts that degraded CT number accuracy. This pipeline included the correction for X-ray scatter, projection data truncation, beam-hardening, and ring-artifact. Methods of each correction step are introduced below.

\section{Scatter correction}

The method of scatter correction used in this study was similar to the one in Reference [12], with the assumption that the detector signal behind the collimator blades was fully induced by X-ray scatter. Specifically, the detector signal measured from collimator shadow was directly used to estimate the scatter fluence outside the primary beam. Measurement was performed in the region of support $Z_{\text {top }}$ and $Z_{\text {bottom }}$ (both covered 50 detector rows, i.e. $16.8 \mathrm{~mm}$ ) (Fig. 1), which was separated from the collimator edges by a small stand-off region $\left(Z_{\text {off }}=18\right.$ detector rows, i.e. $6.05 \mathrm{~mm}$ ) to avoid influence of potential penumbra and extra-focal radiation. Then, 1D scatter profile under the collimator slit was estimated by fitting single Gaussian kernel (Eq. (1)) along each detector column:

$$
\mathrm{S}_{\mathrm{est}}=\mathrm{a} \cdot \exp \left(-\left(\frac{\mathrm{S}_{\text {meas }}-\mathrm{b}}{\mathrm{c}}\right)^{2}\right)
$$

where $S_{\text {est }}$ denotes the estimated 1D scatter profile under the collimator slit at a given detector column, $S_{\text {meas }}$ denotes the measured scatter profile from collimator shadow, and $a, b$ and $c$ denote the kernel parameters that were calculated during fitting. The 1D scatter profile of all detector column formed 2D scatter fluence. Then, the 2D scatter fluence was further smoothed by applying a $2 \mathrm{D}$ median filter (with a square kernel of $13 \times 13$ detector pixels). Finally, the estimated scatter fluence was subtracted from the raw projection measured at each view, to suppress scatter artifact.

\section{Projection data truncation correction}

In MV-FBCT, the scanning FOV did not fully cover the treatment couch. The obvious attenuation of treatment couch outside of FOV caused truncation artifact which contributed to non-uniform CT number bias across the reconstructed images. The reason is briefly explained as 


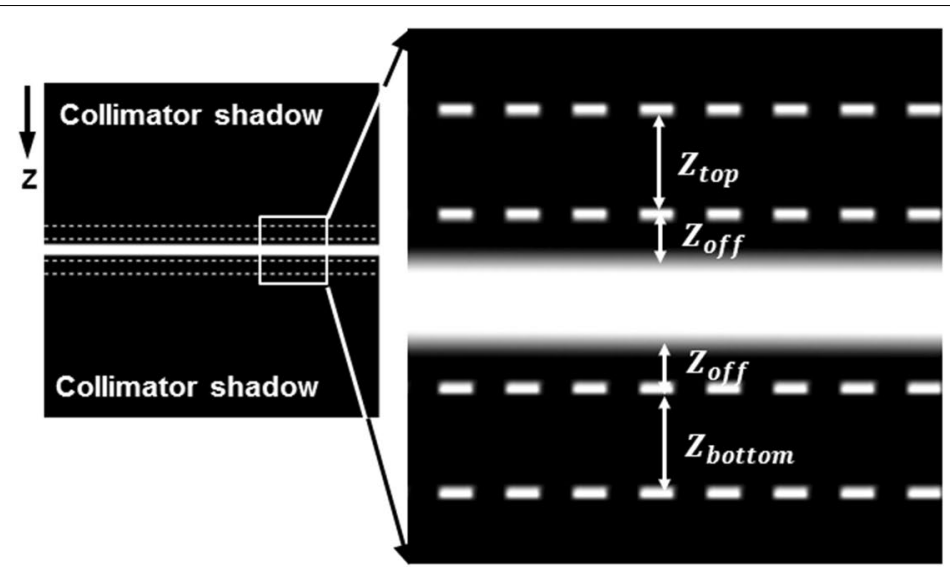

(a)

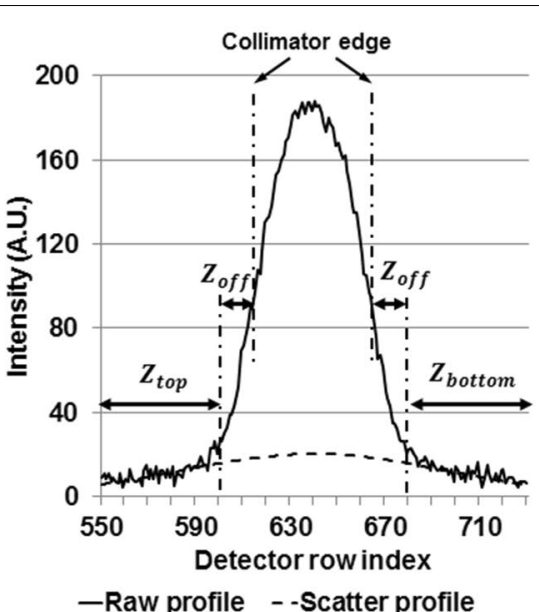

(b)

Fig. 1 a Schematic illustration of region of support that was used in scatter correction. $\mathbf{b}$ The profiles of the measured detector signal (denoted as "Raw profile") and the estimated scatter signal (denoted as "Scatter profile"). The profiles were extracted from the central detector column. $\boldsymbol{Z}_{\text {off }}$ denotes the stand-off region. $\boldsymbol{Z}_{\text {top }}$ and $\boldsymbol{Z}_{\text {bottom }}$ denote the region of support where the measured detector signal was used as scatter fluence

follows. As part of the couch was outside the FOV of MVFBCT, the acquired projection data suffered from truncation (i.e. no measured projection data along rays outside of the FOV) and thus no longer met the data sufficiency condition for the theoretically-exact CT image reconstruction [13-16]. As a result, non-uniform CT number bias was present across the reconstructed FOV, with CT number gradually decreased towards the center of the FOV [15-18]. To correct the truncation artifact, attenuation of treatment couch was measured, without placing phantoms in the scanning FOV. Scatter correction was also conducted in each view as described in Sec. 2.2.1. There existed slight random view offsets (roughly range from $0.1^{\circ}$ to $0.5^{\circ}$ ) between phantom scans and patient couch scan, due to the finite tolerance of gantry rotation. Such view offset induced obvious CT number bias. To overcome this problem, an angular shape-preserving piece-wise cubic interpolation was employed to estimate the patient couch attenuation at exactly the same views in phantom scans. Briefly, the expected signal of each detector pixel at the missing views was interpolated from the measured signal of the same detector pixel across all views acquired in the patient couch scan. Finally, viewwise subtraction was used to remove patient couch attenuation from the measured phantom attenuation.

\section{Beam-hardening correction}

We acquired the projections of a set of solid water slabs with various thickness $(n=11$, thickness ranged from 1 to $35 \mathrm{~cm}$ ) from MV-FBCT, with the primary beam being perpendicular to the water slabs and patient bed. The correction of scatter and projection data truncation was conducted before further processing. Then, the hardened linear attenuation coefficient (LAC) at each solid water thickness was measured by taking the logarithm of the ratio between the measured solid water attenuation and air projection. A 3-degree polynomial regression model was obtained by fitting the hardened LAC over the penetration depth (Fig. 2). The un-hardened LAC (0.1380 1/ $\mathrm{cm}$ ) of solid water was estimated by extrapolating hardened LAC at 0 thickness of solid water using the regression model. The un-hardened solid water attenuation was then calculated by multiplying the un-hardened LAC with varying penetration depth. Further, a secondary 3-degree polynomial regression model was built by fitting the measured solid water attenuation $P_{\text {meas }}$ over the estimated un-hardened solid water attenuation $P_{\text {correct }}$ :

$$
\begin{aligned}
P_{\text {correct }}= & 0.00754 \cdot P_{\text {meas }}^{3}+0.0170 \cdot P_{\text {meas }}^{2} \\
& +1.054 \cdot P_{\text {meas }}-0.0067
\end{aligned}
$$

All measured projection data were processed with Eq. (2) to correct beam hardening effect.

\section{Ring artifact correction}

Ring artifact correction was carried out in the sinogram extracted from each detector row, after the corrections of X-ray scatter, projection truncation, and beam-hardening. For each sinogram, the mean attenuation profile was calculated by averaging the detector signal across all views (Fig. 3a). Then, the mean attenuation profile was smoothed using a moving-average filter with a $7 \times 1$ kernel. The detector anomalies were manifested as the difference between the smoothed and un-smoothed mean attenuation (Fig. 3b). Finally, the detector anomalies were 


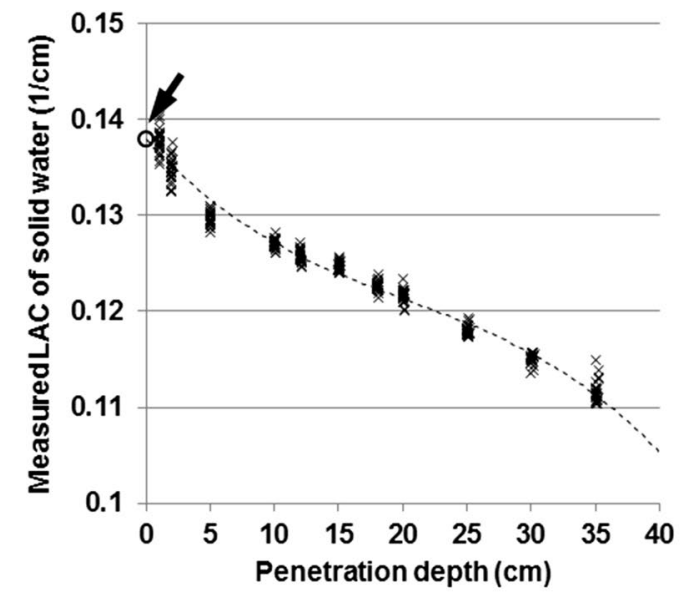

(a)

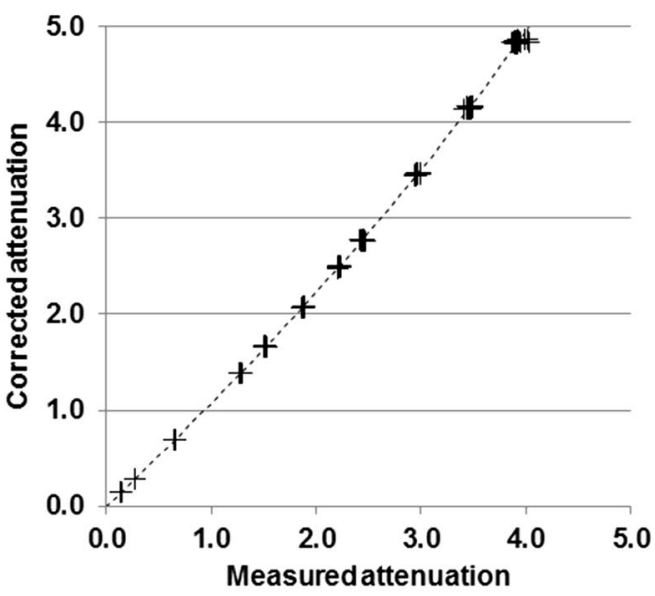

(b)

Fig. 2 a The 3-degree polynomial fitting between the measured linear attenuation coefficient (LAC) and the corresponding penetration depth. The arrow indicates the estimated un-hardened LAC (i.e. $0.13801 / \mathrm{cm}$ ) at $0 \mathrm{~cm}$ penetration depth. $\mathbf{b}$ The 3-degree polynomial fitting between the measured solid water attenuation and the corrected attenuation. The formula of the polynomial model is shown in Eq. (2)

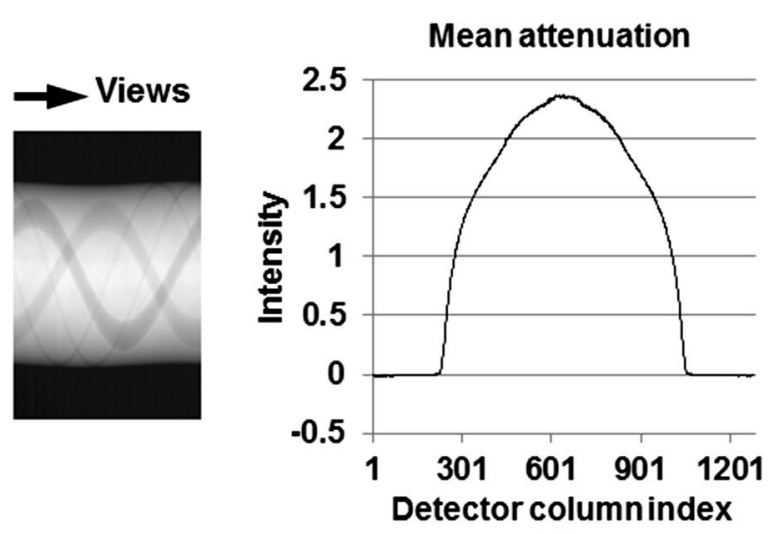

(a)

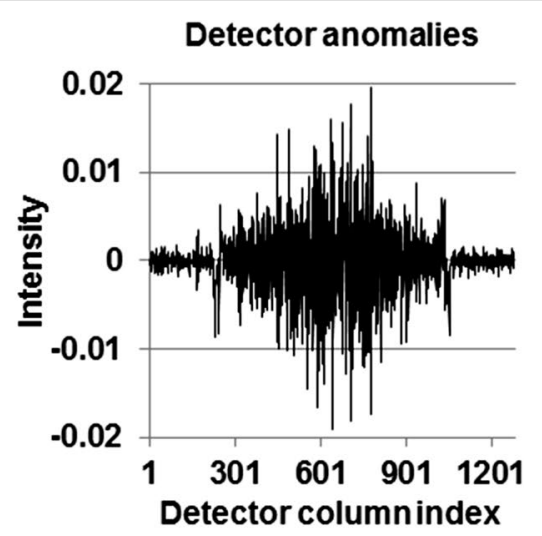

(b)

Fig. 3 The example profiles of mean attenuation (a) and detector anomalies (b) from the sinogram shown in the inset. The mean attenuation was calculated by averaging the measured attenuation along the view direction. The magnitude of detector anomalies was the difference between the mean attenuation and the smoothed mean attenuation

subtracted across all views in each sinogram, to suppress the ring artifact.

\section{Evaluation methods}

We compared the image quality of Catphan phantom before and after applying the in-house artifact corrections. The image quality was evaluated in terms of image artifacts, spatial resolution, CT number bias, uniformity and contrast-to-noise-ratio (CNR). Further, we measured the mean CT numbers of varying Gammex and CIRS inserts in MV-FBCT and TP-CT images, respectively. The accuracy of HU-RED calibration was separately evaluated in the three configurations of insert materials with known REDs (Table 2). Specifically, linear regression between mean CT numbers and REDs was conducted with the interception term fixed at 1.0. The determination coefficient (i.e. $R^{2}$ ) was used to gauge the accuracy of correlation between CT number and RED. The results acquired from TP-CT were used as the baseline to be compared with.

\section{Results}

\section{The effects of artifact correction}

Without preprocessing, MV-FBCT images presented significant cupping artifact and ring artifact (e.g. Catphan images in Fig. 4). Cupping artifact induced non-uniform 


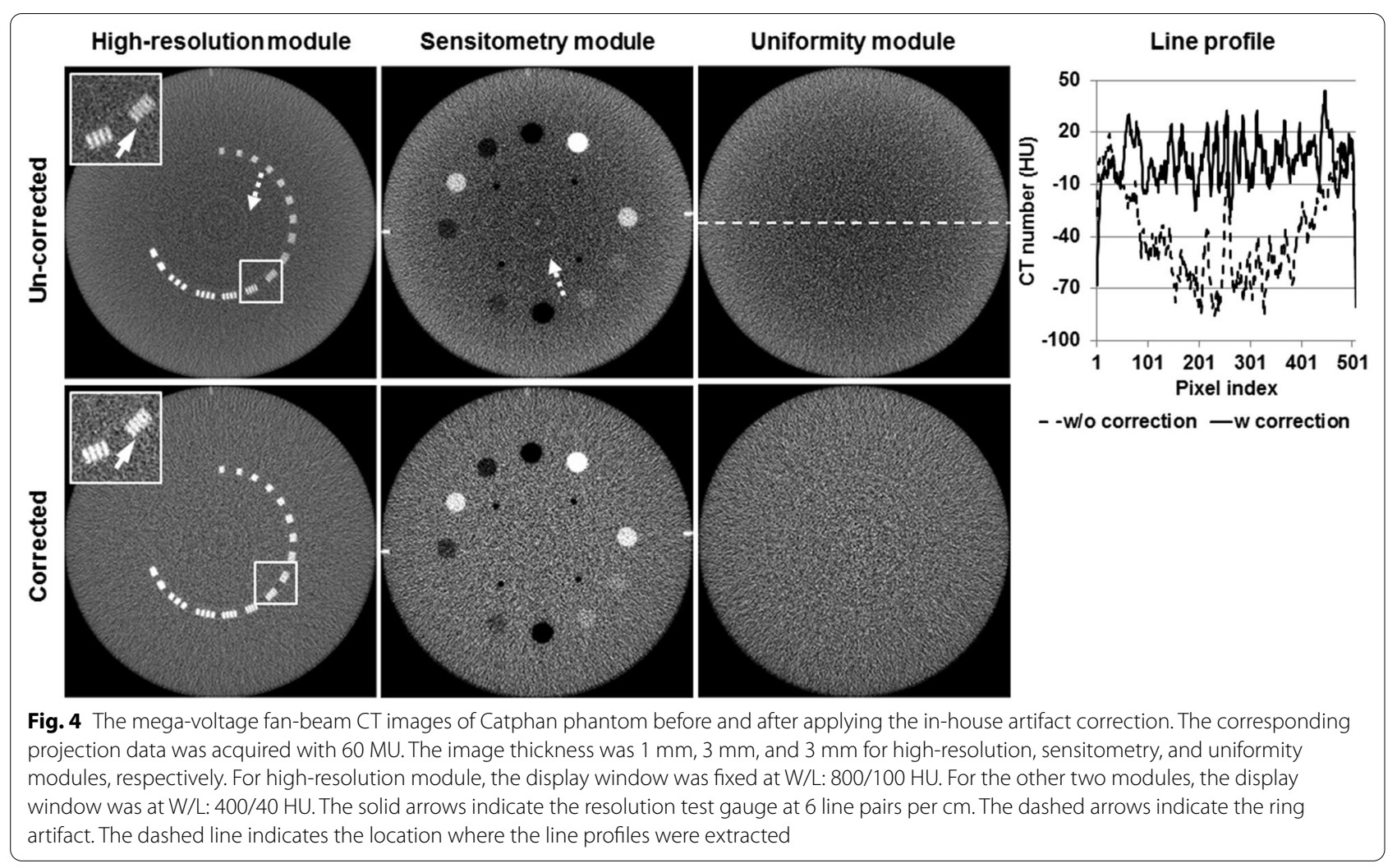

Table 3 Evaluation of image uniformity with Catphan uniformity module: mean $\mathrm{CT}$ number \pm standard error at each region-ofinterest $(\mathrm{ROI})$ *

\begin{tabular}{|c|c|c|}
\hline Dose level & $30 \mathrm{MU}$ & $60 \mathrm{MU}$ \\
\hline Central ROI & $1.7 \pm 2.0 \mathrm{HU}$ & $0.6 \pm 1.2 \mathrm{HU}$ \\
\hline Peripheral ROI \#1 & $1.5 \pm 1.6 \mathrm{HU}$ & $-1.0 \pm 0.9 \mathrm{HU}$ \\
\hline Peripheral ROI \#2 & $1.3 \pm 1.6 \mathrm{HU}$ & $-0.2 \pm 0.9 \mathrm{HU}$ \\
\hline Peripheral ROI \#3 & $-0.4 \pm 1.5 \mathrm{HU}$ & $-0.4 \pm 1.0 \mathrm{HU}$ \\
\hline Peripheral ROI \#4 & $-1.4 \pm 1.6 \mathrm{HU}$ & $-1.1 \pm 1.0 \mathrm{HU}$ \\
\hline
\end{tabular}

${ }^{*}$ In-plane ROI diameter-2.3 cm; slice thickness- $0.3 \mathrm{~cm}$

CT number bias across the entire images. The maximal CT number bias was approximately $70 \mathrm{HU}$ in the uniformity module before artifact correction was applied (see the line profiles in Fig. 4). The in-house artifact correction successfully suppressed image artifacts, and maintained image uniformity and spatial resolution (e.g. the maximal resolution $6 \pm 0.5$ line pairs $/ \mathrm{cm}$ with $1 \mathrm{~mm}$ image thickness). Image uniformity was evaluated with the uniformity module of Catphan phantom, comparing the mean CT number measured from one central regionof-interests (ROI) and four peripheral ROIs (Table 3). Contrast-to-noise-ratio (CNR) was measured with Catphan sensitometry module (Table 4 ).

\section{Correlation analyses}

Compared to TP-CT, MV-FBCT yielded relatively stronger linear correlation between mean $\mathrm{CT}$ numbers and REDs of all insert materials. For soft-tissue inserts, the value of $R^{2}$ was $\leq 0.9990$ in TP-CT but $\geq 0.9994$ in MV-FBCT (Fig. 5). Moreover, we observed that the measured mean CT numbers of Gammex blood-iodine mixture inserts in TP-CT drastically deviated from the fitted linear trendline (see the top left chart in Fig. 5) but not in MV-FBCT. This phenomenon is further discussed in Sec.4. For bone inserts, the value of $R^{2}$ was 0.9950 in TP-CT but $\geq 0.9979$ in MV-FBCT, which may be attributed to less residual beam-hardening artifacts in MVFBCT images. Further, the accuracy of linear correlation between CT numbers and REDs did not decrease at the lower radiation dose level (30 MU) in MV-FBCT (see the charts in the middle column of Fig. 5).

\section{Discussion}

In this study, we implemented MV-FBCT on a commercial LINAC at our institution, using portal imaging functionality and MLC. Then, we developed in-house data preprocessing methods to correct cupping artifact (caused by beam hardening effect and scatter) and CT number bias in MV-FBCT images. Finally, we evaluated 
Table 4 Evaluation of contrast-noise-ratio (CNR) with Catphan sensitometry module*

\begin{tabular}{llll}
\hline & Dose level & $\mathbf{3 0 ~ M U}$ & $\mathbf{6 0}$ \\
\hline & Insert \#1 & 3.7 & \\
\hline \\
\hline
\end{tabular}

* For CNR measurement at each insert, the in-plane ROls were placed at the center of each insert and the immediate background region. ROI diameter-0.8 $\mathrm{cm}$; slice thickness $-0.3 \mathrm{~cm}$. The inset illustrates the indices of inserts

the accuracy of HU-RED calibration and compared to the standard clinical treatment planning $\mathrm{CT}$, using three experimental configurations of different insert materials commonly seen in radiation therapy.

In the evaluation of HU-RED calibration, two Gammex blood-iodine mixture inserts (RED: 1.037 and 1.039) were included to mimic contrast-enhanced soft-tissue (e.g. Thyroid). The corresponding CT numbers from TP-CT obviously deviated from the extrapolated values according to the fitted curve (Fig. 5), and thus additional calibration would be necessary for these materials. This phenomenon was caused by the change of effective atomic number of different materials, which influenced the proportion of Compton scattering and photoelectric effects in TP-CT [19]. Further, other researchers have noted that TP-CT could result in ambiguity in HU-RED calibration curves over the similar RED range (approximately around RED 1.0) [20, 21]. In contrary, MV-FBCT could be employed to avoid such ambiguity and thus further improve calibration accuracy. Moreover, we observed $R^{2}$ value for bone inserts was slightly lower compared to that for soft-tissue inserts, indicating that a stronger beam-hardening correction may be needed for bone inserts in both TP-CT and MV-FBCT. Nevertheless, our experimental results indicated that MV-FBCT yielded stronger linear correlation between $\mathrm{CT}$ number and RED than TP-CT, and provided the potential to use single HU-RED calibration curve for all materials.

In this work, there was no flattering filter with the 2.5 MV beam, and thus the beam-hardening effect would be similar to that in MV-CBCT. Of note, the flattening filter could be used to further suppress beam-hardening artifact, which would be an interesting direction in a follow-up study. Due to larger collimation, MV-CBCT would yield much stronger scatter-to-signal ratio than
MV-FBCT. Given the same incident dose, the scattercorrected MV-CBCT image would tend to present stronger noise compared to MV-FBCT. If one aims to achieve the same image noise level, MV-CBCT would eventually induce higher radiation dose than MV-FBCT. In addition, our current scatter correction method may not be suitable for MV-CBCT, since the accuracy of the estimated scattering signal can decrease as less collimator shadow would be available. This is similar to what was already well-known in $\mathrm{kV}-\mathrm{CBCT}$ [12]. To achieve better outcome, more advanced methods are likely needed for MV-CBCT scatter correction, e.g. the alternating pulse sequence based direct measurement [22]. Furthermore, MV-FBCT would greatly suppress the beam-hardening and metal implant artifacts, especially for the highlyattenuating materials. In contrary, these artifacts can still obviously corrupt DE-CT images, which degrades DE-CT number accuracy [23]. DE-CT has been investigated in a great extent, while MV-FBCT has not been thoroughly investigated.

We acknowledge several limitations in this preliminary study. First, the presented MV-FBCT only provides a sufficient scanning FOV for head/neck scans $(28.7 \mathrm{~cm})$. Truncation caused by patient table was corrected by subtracting projection from pre-scanned table. When MV-FBCT is applied to larger phantoms or body parts, it would be necessary to update the artifact correction methods to address additional projection truncation induced by larger subject size. Second, we did not assess MV-FBCT image quality and HU-RED calibration using dynamic phantoms. In practice, the movement of anatomical structure (e.g. heart and lung) can induce extra CT number bias. This is not a main concern for head and neck applications (the main potential applications due to limited FOV of the MV-FBCT) where there 


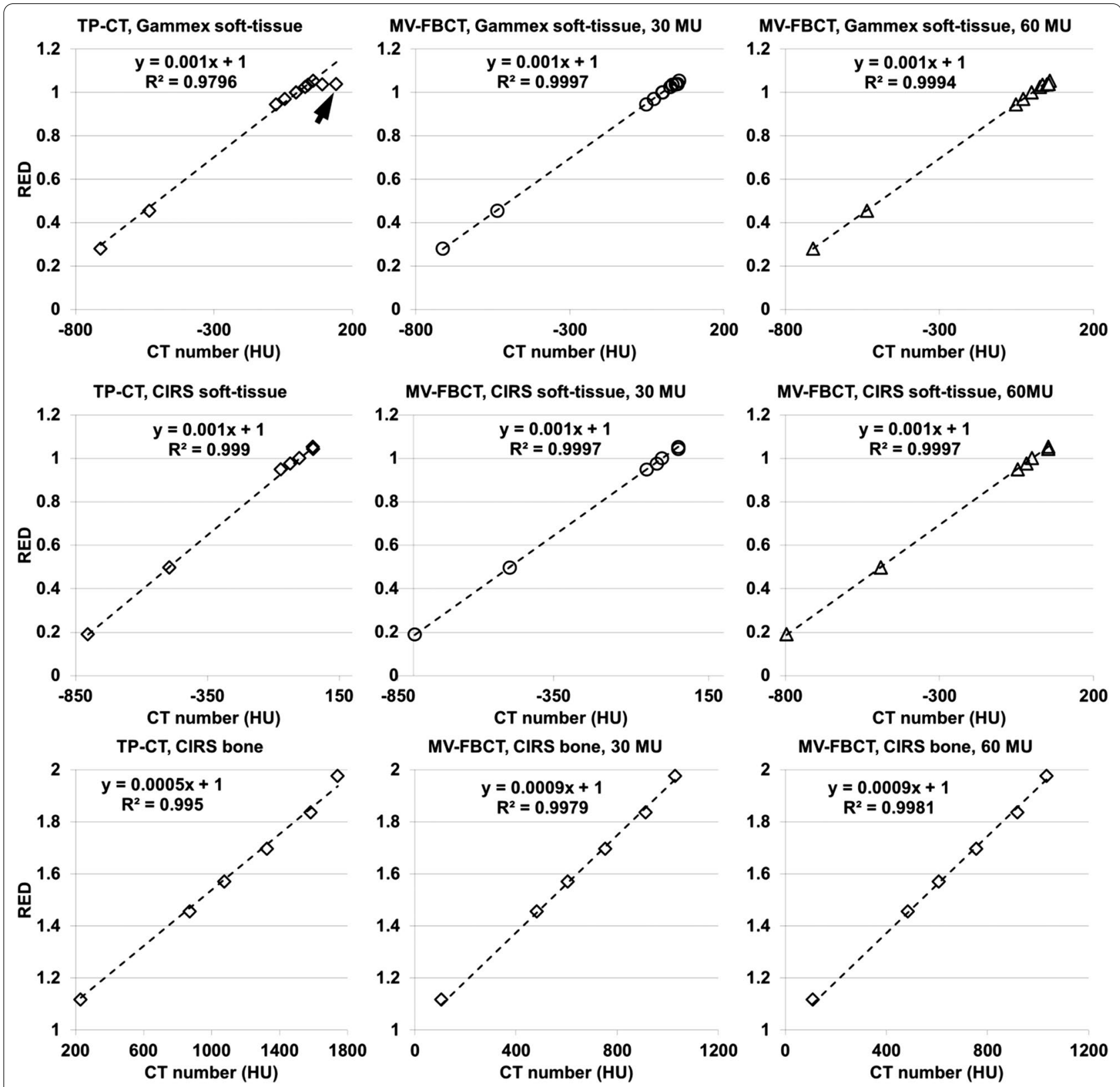

Fig. 5 The linear regression between relative electron densities (RED) and mean CT numbers in clinical CT (TP-CT, with routine radiation dose) and mega-voltage fan-beam CT (MV-FBCT, with $30 \mathrm{MU}$ and $60 \mathrm{MU}$ ). The Gammex soft-tissue inserts (top charts) include adipose, brain, breast, blood, liver, and solid water. The CIRS soft-tissue inserts (bottom charts) include lung (inhale), lung (exhale), adipose, breast, solid water, muscle, and liver. For bone inserts, the corresponding mass densities of ranged from 200 to $1750 \mathrm{mg} / \mathrm{cc}$. The formula of linear regression and the determination coefficient are presented next to the dashed trendline. The solid arrow indicates the points with respect to the blood-iodine mixture inserts

is relatively less patient motion. However, additional motion artifact correction would be mandatory, if MVFBCT is applied in RT to thorax region. Third, we have not investigated the lowest imaging dose level that would be sufficient for dose calculation using MV-FBCT, neither the actual accuracy of dose calculation over anatomical structures. This study mainly focused on the feasibility of performing MV-FBCT using a commercial LINAC. Further studies are warranted to determine the lowest imaging dose and the accuracy of dose calculation over anatomic structures. Image acquisition time is also a limitation of the proposed method of using a LINAC to acquire MV-FBCT images. Since the MV-FBCT only covers $1 \mathrm{~cm}$ width in the superior-inferior $(\mathrm{S} / \mathrm{I})$ direction 
per gantry rotation (typically $1 \mathrm{~min}$ ), MV-FBCT image acquisition time can be significantly longer compared to MV-CBCT or traditional TP-CT simulation for the same coverage. To minimize impact of slow image acquisition, it is recommended to acquire MV-FBCT only around the target area for the purpose of dose calculation and plan optimization. The larger scan range for patient setup and contouring of the entire organs-at-risk will be achieved using traditional TP-CT simulation. Another limitation is imaging dose. In this study, we focused on the feasibility investigation, instead of imaging dose. The dose used in this study is higher compared to $\mathrm{kV} \mathrm{CT} \mathrm{simula-}$ tion. Future studies focusing on minimizing imaging dose while maintaining image quality are warranted. Given the fact that MV-FBCT has long acquisition time and additional dose, use of MV-FBCT requires careful evaluation of risks and benefits. It may not be necessary for every disease sites. But for certain cases where critical structures (e.g. brain stem) are very close to the treatment target, use of MV-FBCT may become justifiable.

\section{Conclusions}

We successfully implemented portal MV-FBCT imaging on a TrueBeam ${ }^{\circledR}$ LINAC that was originally equipped with on-board kV-CBCT. Our in-house artifact correction methods suppressed CT number bias induced by image artifacts. Finally, this in-house-implemented MVFBCT may provide an accurate estimation of electron density distribution, potentially leading to accurate dose calculation for treatment plans in proton therapy.

\section{Abbreviations}

EPID: Electronic portal imaging device; FBCT: Fan-beam CT; FOV: Field-of-view; $\mathrm{HU}$ : Hounsfield unit; LINAC: Linear accelerator; LAC: Linear-attenuation-coefficient; MLCs: Mutlileaf collimators; MU: Monitor unit; MVCT: Mega-voltage CT; MV-CBCT: Mega-voltage cone-beam CT; MV-FBCT: Mega-voltage fan-beam CT; OAR: Organ-at-risk; RED: Relative electron density; RT: Radiation therapy; SPR: Stopping power ratio; TP-CT: Treatment-planning Computed Tomography.
\end{abstract}

\section{Acknowledgements}

We would like to thank Thanos Etmektzoglou from Varian Medical Systems for helping us with programming in developer mode.

\begin{abstract}
Authors' contributions
HG developed the artifact correction methods, evaluated MV-FBCT image quality, analyzed HU-to-RED correlation, and he was a major contributor in writing this manuscript. ST implemented image reconstruction method, and also contributed to writing this manuscript. JDG, WL, and JS were major contributors in developing the methods to acquire MV-FBCT data from LINAC. $\mathrm{CHM}$ was a contributor in revising this manuscript. $\mathrm{YH}$ and $\mathrm{SL}$ designed this study. All authors read and approved the final manuscript.
\end{abstract}

\section{Funding}

This work is supported by the Matteson Funds.
Availability of data and materials

The datasets used and/or analyzed during the current study are available from the corresponding author on reasonable request.

\section{Declarations}

Ethics approval and consent to participate

Not applicable.

\section{Consent for publication}

Not applicable.

\section{Competing interests}

The authors declare that they have no competing interests.

Received: 25 June 2020 Accepted: 19 July 2021

Published online: 28 July 2021

\section{References}

1. Jaffray DA. Image-guided radiotherapy: from current concept to future perspectives. Nat Rev Clin Oncol. 2012;9(12):688-99.

2. Chen GT, Sharp GC, Mori S. A review of image-guided radiotherapy. Radiol Phys Technol. 2009;2(1):1-12.

3. Mackie TR, Holmes T, Swerdloff S, Reckwerdt P, Deasy JO, Yang J, et al. Tomotherapy: a new concept for the delivery of dynamic conformal radiotherapy. Med Phys. 1993;20(6):1709-19.

4. Jaffray DA, Lindsay PE, Brock KK, Deasy JO, Tome WA. Accurate accumulation of dose for improved understanding of radiation effects in normal tissue. Int J Radiat Oncol Biol Phys. 2010;76(3 Suppl):S135-9.

5. Ahnesjö A, Aspradakis MM. Dose calculations for external photon beams in radiotherapy. Phys Med Biol. 1999;44(11):R99-155.

6. Chen W-Z, Xiao Y, Li J. Impact of dose calculation algorithm on radiation therapy. World J Radiol. 2014;6(11):874-80.

7. Thomas SJ. Relative electron density calibration of CT scanners for radiotherapy treatment planning. Br J Radiol. 1999;72(860):781-6.

8. Schneider U, Pedroni E, Lomax A. The calibration of CT Hounsfield units for radiotherapy treatment planning. Phys Med Biol. 1996;41(1):111-24.

9. Knöös T, Nilsson M, Ahlgren L. A method for conversion of hounsfield number to electron density and prediction of macroscopic pair production cross-sections. Radiother Oncol. 1986;5(4):337-45.

10. Hu Y, Ding X, Shen J, Bues M, Liu W, Kang Y, et al. Feasibility of using megavoltage computed tomography to reduce proton range uncertainty: a simulation study. J Appl Clin Med Phys. 2021;22(3):131-40.

11. Newhauser WD, Giebeler A, Langen KM, Mirkovic D, Mohan R. Can megavoltage computed tomography reduce proton range uncertainties in treatment plans for patients with large metal implants? Phys Med Biol. 2008;53(9):2327-44.

12. Siewerdsen JH, Daly MJ, Bakhtiar B, Moseley DJ, Richard S, Keller H, et al. A simple, direct method for $x$-ray scatter estimation and correction in digital radiography and cone-beam CT. Med Phys. 2006;33(1):187-97.

13. Tuy HK. An Inversion Formula for Cone-Beam Reconstruction. SIAM J Appl Math. 1983;43(3):546-52.

14. Smith BD. Image reconstruction from cone-beam projections: necessary and sufficient conditions and reconstruction methods. IEEE Trans Med Imaging. 1985:4(1):14-25.

15. Tang $X$, Krupinski EA, Xie H, Stillman AE. On the data acquisition, image reconstruction, cone beam artifacts, and their suppression in axial MDCT and CBCT-a review. Med Phys. 2018;45(9):e761-82.

16. Wang $\mathrm{G}, \mathrm{Yu} \mathrm{H}$. The meaning of interior tomography. Phys Med Biol. 2013;58(16):R161-86.

17. Gong H, Li B, Jia X, Cao G. Physics model-based scatter correction in multi-source interior computed tomography. IEEE Trans Med Imaging. 2018;37(2):349-60. 
18. Zou Y, Pan X, Sidky EY. Image reconstruction in regions-of-interest from truncated projections in a reduced fan-beam scan. Phys Med Biol. 2004;50(1):13-27.

19. Khan FM. The Physics of Radiation Therapy: Lippincott Williams \& Wilkins; 2010

20. Das IJ, Cheng C-W, Cao M, Johnstone PAS. Computed tomography imaging parameters for inhomogeneity correction in radiation treatment planning. J Med Phys. 2016;41(1):3-11.

21. Cheng C-W, Zhao L, Wolanski M, Zhao Q, James J, Dikeman K, et al. Comparison of tissue characterization curves for different $C T$ scanners: implication in proton therapy treatment planning. Transl Cancer Res. 2013;1 (4):236-46

22. van Herk M, Ploeger $L$, Sonke JJ. A novel method for megavoltage scatter correction in cone-beam CT acquired concurrent with rotational irradiation. Radiotherapy Oncol J Eur Soc Therapeutic Radiol Oncol. 2011;100(3):365-9.

23. Patrick JC, Terry Thompson R, So A, Butler J, Faul D, Stodilka RZ, et al. Technical Note: comparison of megavoltage, dual-energy, and single-energy CT-based $\mu$-maps for a four-channel breast coil in PET/MRI. Med Phys. 2017;44(9):4758-65.

\section{Publisher's Note}

Springer Nature remains neutral with regard to jurisdictional claims in published maps and institutional affiliations.
Ready to submit your research? Choose BMC and benefit from:

- fast, convenient online submission

- thorough peer review by experienced researchers in your field

- rapid publication on acceptance

- support for research data, including large and complex data types

- gold Open Access which fosters wider collaboration and increased citations

- maximum visibility for your research: over $100 \mathrm{M}$ website views per year

At BMC, research is always in progress.

Learn more biomedcentral.com/submissions 